Journal of

Accident and

Emergency

Medicine 1995

12, 43-48

\title{
Heroin bodypacking
}

\author{
P.J.LEO, ${ }^{1}$ J.J.SACHTER ${ }^{2} \&$ M. MELROSE ${ }^{3}$
}

${ }^{1}$ Mount Sinai School of Medicine Integrated Residency in Emergency Medicine, New York

2 Department of Emergency Medicine, Elmhust Hospital Center, Elmhurst, New York

${ }^{3}$ Division of Emergency Medical Services, Department of Medicine, Beth Israel Medical Center, New York

\section{SUMMARY}

Drug smuggling by internal bodily concealment is a well-recognized international problem, particularly in view of the difficulty of its detection and the potential for large financial gains. This mode of transport can have serious medical complications, including drug intoxication - sometimes fatal - as well as intestinal obstruction by foreign bodies (FBs). We discuss a case of heroin 'bodypacking' (the ingestion of heroin filled condoms) with its resultant complications. The initial medical management and indications for surgery are discussed, and the relevant literature reviewed.

Key words: bodypacker, bodystuffer, heroin, naloxone

\section{INTRODUCTION}

The use of the human gastrointestinal (GI) tract to smuggle drugs is a recognized problem in many major cities. ${ }^{2,3,25}$ Although medical attention is not sought in the majority of cases, the 'mule', or 'bodypacker' may present with life-threatening symptoms of intoxication including seizures, cardiopulmonary collapse, as well as mechanical obstruction from the ingested drug packets. ${ }^{5,24}$ The first reported case of intoxication related to drug smuggling via the GI tract - ingestion of balloons filled with cocaine - was described by Mebane \& DeVito in $1975 . .^{18}$ In 1980, Wetli \& Mittleman described the 'bodypacker syndrome'. 32 They reported fatalities in 10 out of 11 cases occurring in travellers returning to the United States on flights from South America. All of the victims had swallowed bags or condoms filled with cocaine to avoid detection by US Customs officials, hence the term 'bodypacking'. Mortality in cases reported up to 1981 was greater than $50 \% .^{10}$ Since then, there have been an increasing number of reports of cocaine bodypacking. There have been far fewer cases involving the smuggling of heroin via the GI tract reported in the medical literature. ${ }^{3,7,10,11,25,29,31}$ Review of the English language acute care literature to date reveals only one report of heroin overdose via this method of transport. ${ }^{31}$ To date, a consensus on the management of these cases has not emerged. Previous cases have reported radiographic and toxicological findings but no comprehensive management strategy. ${ }^{3,27}$ We present such a case and suggest guidelines regarding the management of possible complications in such patients.

\section{CASE REPORT}

History

A 31-year-old Hispanic male was brought to the Emergency Department (ED) at Elmhurst Hospital Center by taxi from a local airport. The driver stated that his passenger had become very drowsy. Upon presentation to the ED, the patient was unresponsive to both verbal and painful stimuli. Respirations were noted to be shallow and irregular. A peripheral intravenous line was quickly established and blood was taken for routine laboratory studies. Thiamine $(100 \mathrm{mg}), 50 \mathrm{~mL}$ of $50 \%$ dextrose, and $0.8 \mathrm{mg}$ of naloxone were administered intravenously, and the patient promptly responded. He stated that he had just arrived from Colombia after visiting friends. He denied any history of drug abuse or past medical problems. The patient stated that he became drowsy from paint fumes in the back seat of the taxi and that he did not recall anything else.

\section{Physical examination}

Initial physical examination revealed an obtunded, slightly diaphoretic male. His vital signs were: blood pressure of $184 / 85 \mathrm{mmHg}$; temperature $99.4 \mathrm{~F}$ (orally); pulse 88 and regular; and respirations approximately eight per minute, irregular and shallow.

(C) 1995 Blackwell Science Ltd 
HEENT examination revealed bilateral pinpoint pupils that were unresponsive to light. The neck was supple, lungs clear, and the cardiac exam was normal. Decreased bowel sounds were noted on the abdominal examination. Rectal and genitourinary examinations were unremarkable. Inspection of the extremities revealed no cyanosis, oedema or stigmata of drug abuse.

\section{Emergency department course}

Immediately after the administration of the intravenous thiamine, naloxone, and dextrose, the patient became extremely combative. His heart rate increased to 120 , respiratory rate to 24 , and pupils were now 2 to $3 \mathrm{~mm}$ bilaterally and reactive. The patient wanted to leave the hospital, stating that he felt perfectly fine. A hospital hold was requested at this point and the patient was restrained.

One hour after initial presentation, the patient again became unresponsive and cyanotic. He received another $0.8 \mathrm{mg}$ of naloxone, to which he again responded. At this time, a naloxone infusion was started intravenously at approximately 0.8 $\mathrm{mg} \mathrm{h}^{-1}$. Three hours later, the patient again became unresponsive and cyanotic, despite the continued naloxone infusion and another $0.8 \mathrm{mg}$ bolus injection. The patient was orotracheally intubated, and placed on a volume-cycled respirator at 16 breaths per minute. His physical examination remained essentially unchanged, with a persistent miosis and variable respirations.

Laboratory studies revealed a peripheral leucocyte count of $27500 \mathrm{~mm}^{3}$ (90\% PMNs); Hematocrit was 48 , and the platelet count was normal. Serum electrolytes, including glucose (obtained before giving dextrose), blood urea nitrogen, and creatinine were normal, as were coagulation studies. The urine dipstick (Ames Multistix 9) was positive only for glucose (trace). Serum creatinine phosphokinase (CPK) was $329 \mathrm{UL}^{-1}$ (normal range, 26 to $189 \mathrm{U}$ $\mathrm{L}^{-1}$ ) and was sent for isoenzyme fractionation. $A$ 12-lead electrocardiogram revealed sinus tachycardia without ischaemic changes. PR, QRS, and QT duration were all within normal limits. Subsequent urine and serum toxicology revealed the presence of opiates.

Although the first portable radiographs of the chest (AP) and abdomen were initially interpreted as unremarkable, a review of the original radiographs indicated possible FBs beneath the left hemidiaphragm (see Fig. 1). Repeat radiographs of the chest (AP) and abdomen (flat) after endotracheal

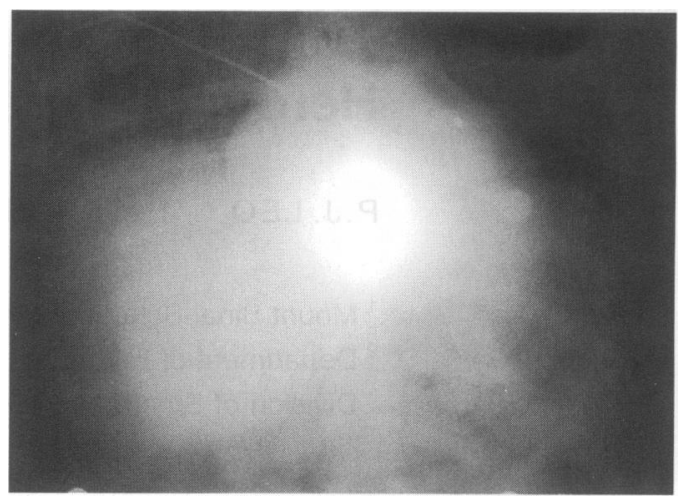

Fig. 1. Abdominal (flat) radiograph showing multiple tubular foreign bodies on the left side of the abdomen.

intubation, were of a better quality, and showed a paucity of intestinal gas with multiple blade-shaped objects in the stomach and colon. The endotracheal tube was in good position. Though the patient continue to deny drug use, it was believed that he had ingested large amounts of narcotic packages. A surgical consultation was obtained and the patient was taken to the operating room.

\section{Subsequent course}

On laparotomy, multiple FBs were palpated in the stomach. Thirty-five $1 \times 5^{\prime \prime}$ drug filled condoms (see Fig. 2) were extracted from the stomach via gastrotomy. The entire bowel was examined and multiple FBs were found in the descending and sigmoid colon (see Fig. 3). These FBs were milked downward to the area of the rectum. Fourteen more drug-filled condoms were removed under direct visualization via anoscopy. Several of the condoms appeared to be broken and in pieces. The patient had a post-operative course complicated by a presumed right aspiration pneumonitis. He was treated prophylactically with intravenous cefazolin and metronidazole, and was extubated on postoperative day 2 with return of bowel function 4 days later. The drug within the condoms was identified by United States Drug Enforcement Agency as 'high grade heroin'. The patient was transferred to a correctional facility on post-operative day 11 in a stable condition.

Because of the elevated CPK measurement in the ED, a repeat CPK measurement $8 \mathrm{~h}$ after the first assay revealed a total CPK of $188 \mathrm{UL}^{-1}$ with negative CPK-MB fractions. The leucocyte count returned to normal. Blood and urine cultures showed no bacterial growth at the time of discharge. 
Heroin bodypacking

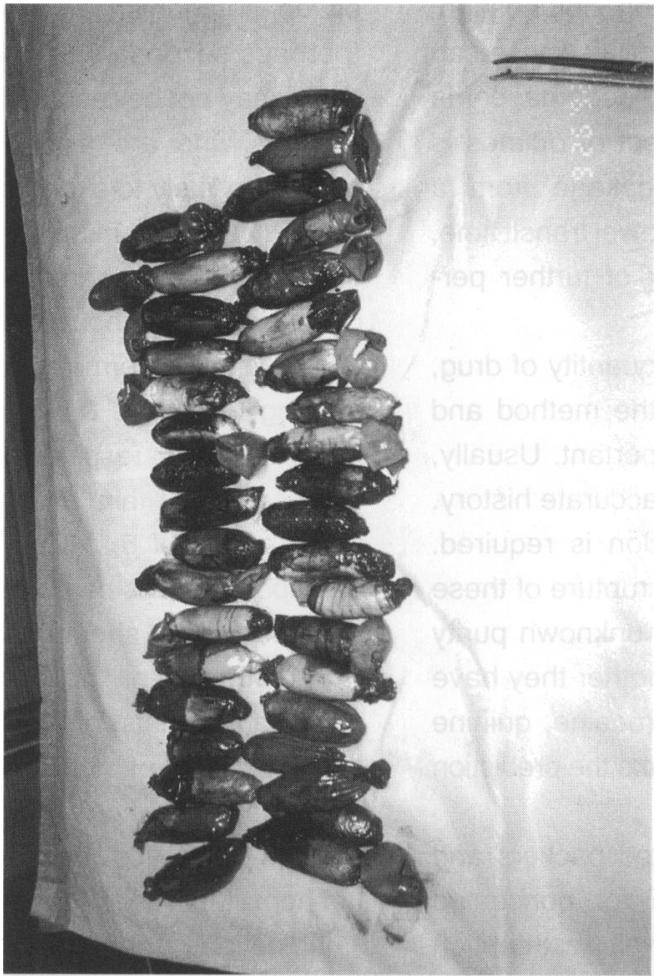

Fig. 2. Thirty-five heroin-filled condoms recovered postoperatively from the stomach.

\section{DISCUSSION}

The patient described was a 'bodypacker', as opposed to a 'bodystuffer'. This distinction was first made by Roberts, et al. in 1985. ${ }^{21}$ Bodypackers are hired specifically to smuggle illegal drugs, usually cocaine or heroin, which are packaged and wrapped in containers that are designed to survive passage through the GI tract. The risk of leaching or package perforation, despite the sophistication of the wrap- ping, appears to increase in proportion to the number of packages ingested. ${ }^{20}$ The bodypacker typically has a history of international travel originating in a country known for drug exportation. Bodypackers present to hospitals because they have developed complications, such as drug intoxication or intestinal obstruction; or occasionally following sudden collapse and subsequent cardiac arrest.

The bodystuffer, on the other hand, swallows poorly packaged - or even unwrapped-contraband to conceal its presence during apprehension. The typical bodystuffer is a user or seller of these illicit substances who, on the verge of arrest, swallows the evidence. The quantity of these substance is generally small when compared with bodypackers. Because of this poor wrapping, lethal exposure may occur despite the relatively smaller quantities ingested. Another significant difference between stuffers and packers is that stuffers are much more likely to be exposed to co-ingestants which can complicate their management. Symptoms and entire toxidromes may vary widely or overlap because of the presence of these co-ingestants. This is because the 'stuffer' is frequently a polysubstance abuser, as opposed to the bodypacker who may not be a drug user at all.

Heroin is absorbed well from the gastrointestinal tract $^{27}$ and its usual duration of action is $3-4 \mathrm{~h}$. The drug is cleared rapidly from plasma and metabolized in the liver, with $90 \%$ excreted in the urine within $24 \mathrm{~h}$. Tolerance develops rapidly with increased use, but there is wide individual variation in sensitivity to the drug. Consequently the acute lethal dose varies from person to person and within individuals depending upon their previous exposure to opiates. Signs and symptoms of opiate toxicity can include

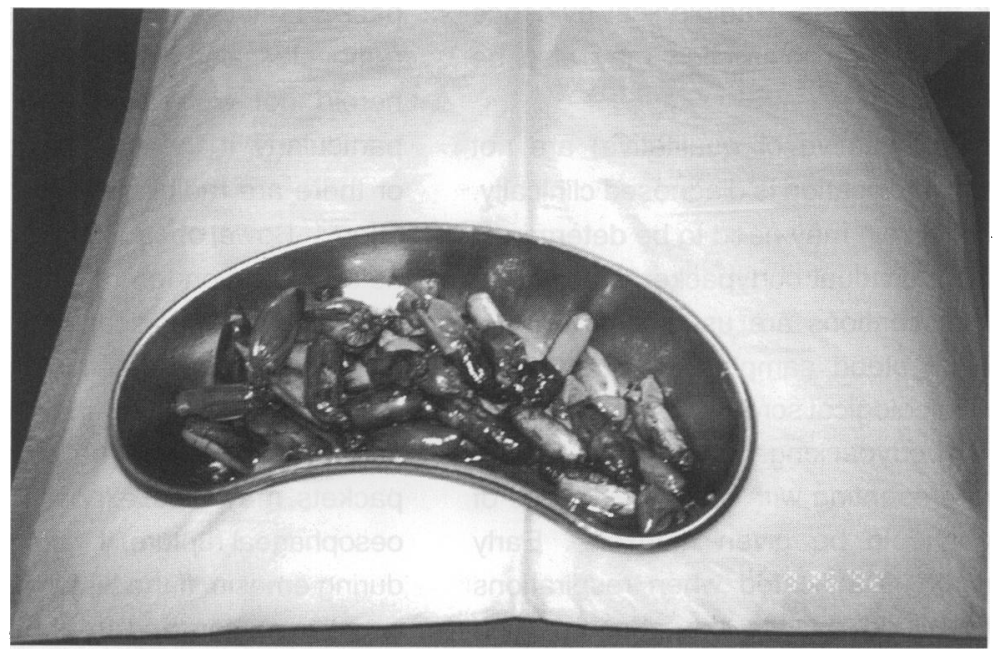

(C) 1995 Blackwell Science Ltd, Journal of Accident and Emergency Medicine 12, 43-48
Fig. 3. Multiple heroin filled condoms recovered postoperatively from the descending and sigmoid colon. Note some of the condoms have ruptured. 
P.J. Leo et al. nausea, vomiting, miosis, constipation, obtundation, respiratory depression and compromise. These can result in non-cardiogenic pulmonary oedema, coma and death. The anticholinergic effect of opiates is an important consideration, as leakage from a swallowed package may lengthen bowel transit time, increasing absorption and the risk of further perforation of packaging materials. ${ }^{27}$

In taking a history, the type and quantity of drug, the type of packaging used, and the method and duration of concealment are all important. Usually, the bodypacker will not provide an accurate history. Therefore, a high index of suspicion is required. Unknown factors include the risk of rupture of these packages, variable GI transit time, unknown purity of the substances ingested, and whether they have been 'cut' with adulterants such procaine, quinine and barbiturates. All these factors make the prediction of clinical outcome difficult.

Physical examination of suspected packers and stuffers should include a search for concealed packages in all body cavities as well as attention to the signs of drug intoxication and intestinal obstruction. The investigation of the suspected bodypacker must include plain abdominal and chest radiographs which may help identify concealed packages. The drug packets may have a radiolucent halo appearance on radiological examination, indicating deterioration of the package with an increased risk of rupture. ${ }^{27}$ The key to detecting these FBs via radiography is the trapping of air in the package, providing an air-fluid or air-foreign object interface. 4,19 The stomach and ascending colon are the most common locations in which FBs are detected because of the contrast afforded by gastric and colonic air that surround the objects. ${ }^{20}$ Foreign bodies in the rectosigmoid colon are often difficult to detect because of the presence of stool that can mask the packets. Radiological evidence of small or large bowel obstruction may also be present.

Drug levels (quantitative or qualitative) are not required if heroin intoxication is diagnosed clinically. The presence of heroin may need to be determined analyticaly if the individual bodypacker is asymptomatic. Such investigations are usually carried out on urine and not blood samples. ${ }^{7,9}$ Note that a negative urine toxicological screen does not exclude the possibility of bodypacking. ${ }^{31}$

Bodypackers presenting with clinical evidence of opiate toxicity should be given naloxone. Early ventilatory support is indicated when respirations become compromised despite the administration of the antagonist, as those with huge overdoses (perhaps secondary to packet rupture), or coingestants, may not be reversed adequately by naloxone. Patients who are also physically addicted to an opioid are likely to experience a precipitous onset of withdrawal within minutes to hours of administration of naloxone, and aggressive treatment of withdrawal is appropriate. Although the exact dose of naloxone has not been determined, we suggest an initial bolus of $2 \mathrm{mg}$, in $0.4-$ to $0.8-\mathrm{mg}$ aliquots. If no response in pupillary size, respiration or level of consciousness is observed within minutes, additional naloxone, up to a total of $5-10 \mathrm{mg}$, may be administered. If unresponsiveness continues, the diagnosis of opiateinduced toxicity should be questioned.

The treatment of heroin induced pulmonary oedema consists of ventilatory support and the administration of naloxone. Other components of cardiogenic pulmonary oedema therapy, such as digitalis, diuretics and rotating tourniquets, are neither effective or necessary. ${ }^{26}$

Indications for surgical intervention are controversial. Since there is no effective, safe cocaine antagonist, laparotomy and removal of leaking packages should be performed as soon as possible after resuscitation and identification of the symptomatic patient as a cocaine bodypacker. Heroin intoxication can and should initially be managed with intravenous naloxone. Repeated episodes of hypotension or apnoea despite naloxone infusion suggest leakage or possibly repture of additional heroin packets. The efficacy of even large doses of naloxone in antagonizing potentially huge amounts of extremely potent (often uncut) narcotics released directly into the GI tract is unclear. We would suggest that continued or intermittent symptomatology in the face of large doses of naloxone $(5-10 \mathrm{mg})$ is one indication for surgical intervention. Since most packets passes spontaneously within $30 \mathrm{~h}^{1}$ surgical removal is also advised for unruptured packets of heroin not excreted within 5 days of ingestion, particularly if there is failure to progress distally or there are radiological signs of package deterioration. ${ }^{27}$ Bowel obstruction is also generally regarded as an indication for immediate laparotomy.

There is no indication for use of ipecac with bodypackers since the history of ingestion is usually greater than $60 \mathrm{~min}$ and the packets have often progressed beyond the stomach. The size of the packets may also expose the patient to a risk of oesophageal rupture, if they obstruct the oesophagus during emesis. If the history is consistent with a truly acute ingestion and the bodystuffer is awake, has 
Heroin

bodypacking not had a seizure, and gives a reliable history of ingestion within $60 \mathrm{~min}$ of arrival, ipecac may be given. ${ }^{6,14,15,21}$

Gastric lavage in both the asymptomatic and symptomatic patient is contraindicated because of the possibility of rupturing a previously intact package, the risk of aspiration, encountering FBs that are too large for the lavage tube, or propelling the packets into the small bowel where they are more likely to cause a mechanical obstruction. Activated charcoal with a cathartic should be given initially. Whole bowel irrigation, administering large volumes of polyethylene glycol (PEG) via naso-gastric tube, is another potential treatment modality. Whole bowel irrigation can be initiated at 1.5-2 $\mathrm{Lh}^{-1}$, and the rectal effluent examined for FBs and the appearance of charcoal from above. ${ }^{8,12}$ A follow-up barium swallow is warranted to visualize the entire GI tract for any leftover packages. Multiple dose activated charcoal therapy is probably contraindicated when combining whole bowel irrigation (WBI). Several authors have reported that the efficacy of activated charcoal is markedly reduced by PEG. ${ }^{12,22,30}$ Utecht, et al. reported one case in which PEG solution 'dissolved' the heroin out of the packages (overwrapped only with electrician's tape) causing further toxicity and complications. ${ }^{31}$ Despite the indications for surgery discussed above, we continue to support the use of WBI/PEG in the nonsurgical management of asymptomatic heroin bodypackers, at least until there are further reports of such complications with the procedure. To date, we have not encountered these complications while using WBI with PEG in bodypackers, either at our institutions, or in discussions with our regional poison centre. It should be noted that the majority of our patients have used latex inner or outer wrappings in packaging their contraband. It is possible in the case reported by Utecht et al. that there may have been multiple leakages through the adhesive material in the electrician's tape used by the patient.

The use of osmotic laxatives, such as lactulose, does help in the expulsion of drug packages from the GI tract but provides no assurances that the packages will not leak or rupture in transit. Stimulant laxatives should be avoided as they may precipitate obstruction. Oral liquid paraffin and arachis oil enemas should not be used as they effect deterioration of the latex rubber often used to package the drugs. ${ }^{27}$ Endoscopic removal of the packages is also not recommended since the possibility of rupturing them exists. ${ }^{3}$

In conclusion, we have presented a case of a symptomatic patient who attempted to transport heroin via the Gl tract. To date, there is only one report in the English language acute care literature of heroin bodypacking. Likewise, there is no consensus on how to manage either asymptomatic and symptomatic heroin bodypackers. Previous suggestions regarding management have been less than comprehensive in scope. We have attempted to provide some more difinitive guidelines for the management of these patients.

\section{REFERENCES}

1. Caruana D.S., Weinbach B. \& Georg D. (1984) Cocaine-packet Ingestion. Annals of Internal Medicine 100, 73-74.

2. Daimant-Berger O, Gherardi R. \& Baud F. (1988) Intracorporeal concealment of narcotics - experience at the medicojudicial emergency centre of the HotelDien Hospital: one hundred cases. Presse Medicin 17, 107-110.

3. Dunne J.W. (1983) Drug smuggling by internal bodily concealment. Medical Journal of Australia 2, 436-439.

4. Fainsinger M.H. (1977) Unusual foreign bodies in bowel. Journal of the American Medical Association 237, 2225-2226.

5. Freed T.A., Sweet L.N. \& Gauder P.J. (1976) Balloon obturation bowel obstruction: A hazard of drug smuggling. Americal Journal of Roentgenology 127, 1033-1034.

6. Freedman G.E., Pasternak S. \& Krenzelok E.P. (1987) A clinical trial using syrup of ipecac and activated charcoal concurrently. Annals of Emergency Medicine 16, 164-166.

7. Gherardi R.K., Leporc P. \& Dupeyron J.P. (1988) Detection of drugs in the urine of bodypackers. Lancet 1, 1076-1077.

8. Hoffman R., Smilkstein M. \& Goldfrank L. (1990) Whole bowel irrigation and the cocaine body-packer: a new approach to a common problem. Americal Journal of Emergency Medicine 8(6), 523-527.

9. Huizer H. (1983) Analytical Studies on Illicit Heroin: I. The Occurrence of O3-Monoacetylmorphine. Journal of Foresic Science 28, 32-39.

10. Introna F. \& Smialek J.E. (1989) The 'mini-packer' syndrome - fatal ingestion of drug containers in Baltimore, Maryland. American Journal of Forensic and Medical Pathology 10(1), 21-24.

11. Joynt B.F. \& Mikhael N.Z. (1985) Sudden death of a heroin body-packer. Journal of Analytical Toxicology 9, 238-240.

12. Kirshenbaum L.A., Sitar D.S. \& Tenenbein M. (1990) Interaction between whole-bowel irrigation solution and activated charcoal: implications for the treatment 


\section{P.J. Leo et al.}

of toxic ingestions. Annals of Emergency Medicine 19, 1129-1132.

13. Lancashire M.J.R., Legg P.K. \& Lowe M. (1988) Surgical aspects of international drug smuggling. British Medical Journal 296, 1035-1037.

14. Linden O.H. \& Rumack B.H. (1984) lpecac for ingested drug packets (abstract). Veterinary and Human Toxicology 26, 404.

15. Marc B., Gherardi R.K. \& Baud F.J. (1989) Managing drug dealers who swallow the evidence. British Medical Journal 299, 1082.

16. Marc B., Gherardi R.K. \& Baud F.J. (1989) The spectrum of in-body drug concealment: diagnosis and emergency treatment. European Journal of Emergencies 2, 81-87.

17. McCarron M.M. \& Wood J.D. (1983) The cocaine 'body-packer' syndrome - diagnosis and treatment. Journal of the American Medical Association 250, 1417-1420.

18. Mebane C. \& DeVito J. (1975) Cocaine intoxication a unique case. Journal of the Florida Medical Association 62, 19-20.

19. Pamilo M., Suoranta H. \& Suramo I. (1986) Narcotic smuggling and radiography of the GI tract. Acta Radiology Diagnostica 27, 213-216.

20. Pollack C., Biggers D. \& Carlton F. (1992) Two crack cocaine body stuffers. Annals of Emergency Medicine 21, 1370-1380.

21. Roberts J.R., Price D. \& Goldfrank L. (1986) The bodystuffer syndrome: a clandestine form of drug overdose. American Journal of Emergency Medicine 4(1), 24-27.

22. Rosenberg P.J. (1988) Effect of whole bowel irrigation on the antidotal efficacy of oral activated charcoal.
Annals of Emergency Medicine 17, 681-683.

23. Schneider V. \& Klug E. (1979) Todlicher heroinschmuggel. Deutsche Med Wochenschr 104, 1282-1283.

24. Simson L.R. (1976) Sudden death while attempting to conceal illegal drugs: laryngeal obstruction by a package of heroin. Journal of Forensic Science 21, 378-380.

25. Sinner W. (1981) The gastrointestinal tract as a vehicle for drug smuggling. Gastrointestinal Radiology 6 , 319-323.

26. Sternbach G.L. (1992) Narcotics, In: Tintinalli J.E., Krome R.L. \& Ruiz E. (eds) Emergency Medicine - A Comprehensive Study Guide, pp. 556-559. McGrawHill, Inc., New York.

27. Stewart A., Heaton N.D. \& Hogbin B. (1990) Bodypacking - a case report and review of the literature. Postgraduate Medical Journal 66, 659-661.

28. Suarez C., Arango A. \& Lester J.L. (1977) Cocainecondom ingestion - surgical treatment. Journal of the American Medical Association 238, 1391-1392.

29. Taudberg D. \& Abercrombie D. (1982) The treatment of heroin overdose with endotracheal naloxone. Annals of Emergency Medicine 11, 443-445.

30. Tenenbein M. (1989) Whole bowel irrigation and activated charcoal (letter). Annals of Emergency Medicine 18, 707.

31. Utecht MJ., Stone A.F. \& McCarron M.M. (1993) Heroin body packers. Journal of Emergency Medicine 11, 33-40.

32. Wetli C.V. \& Mittleman R.E. (1981) The 'bodypacker syndrome' - toxicity following ingestion of illicit drugs packaged for transportation. Journal of Forensic Science 26, 492-500. 